\title{
Modeling carbon emissions from urban land conversion: gamma distribution model
}

\author{
A. Svirejeva-Hopkins ${ }^{1} \&$ H.-J. Schellnhuber ${ }^{2}$ \\ ${ }^{1}$ SIM, Faculty of Science, University of Lisbon, Portugal \\ ${ }^{2}$ PIK Potsdam, Germany
}

\begin{abstract}
In this paper we examine the method used for calculating regional urban area dynamics and the resulting carbon emissions (from the land-conversion only) for the period 1980-2050 for the eight world regions. This approach is based on the fact that the spatial distribution of population density is close to the two-parametric gamma-distribution. The developed model provides us with the scenario of urbanization, based on which the regional and world dynamics of carbon emissions and export from cities, and the annual total urban carbon balance is estimated. According to our estimations, world annual emissions of carbon as a result of urbanisation increased to $1.25 \mathrm{GtC}$ in 2005 and began to decrease afterwards. If we compare the emission maximum with the annual emission caused by deforestation, $1.36 \mathrm{GtC}$ per year, then we can say that the role of urbanised territories (UT) in the Global Carbon Balance is of a comparable magnitude. Regarding the world annual export of carbon from UT, we observe its monotonous growth by three times, reaching $505 \mathrm{MtC}$. The latter is comparable to the amount of carbon transported by rivers into the ocean (196-537 MtC). The current model shows that urbanization is inhibited in the interval 2020-2030, and by 2050 the growth of urbanized areas would almost stop. Hence, the total balance, being almost constant until 2000, then starts to decrease at an almost constant rate. By the end of the XXI century, the total carbon balance will be equal to zero, with the exchange flows fully balanced, and may even be negative, with the system beginning to take up carbon from the atmosphere, i.e., becomes a "sink".
\end{abstract}

Keywords: urban area, carbon emissions, cities' growth, urbanisation, population density, distribution, carbon source and sink, vegetation, carbon cycle. 


\section{Introduction}

In this paper we continue to consider how the urbanisation process influences the Global Carbon Cycle (GCC), using the model of urban area growth based on the two-parametric gamma-distribution of population density (Kendall and Stuart [1], Vaughn [9]). Note that we shall not take into account the clear role of urbanisation in all anthropogenic emissions of $\mathrm{CO}_{2}$. We are calculating the emissions from the conversion of natural ecosystems and landscapes unto urban lands that take place when cities are sprawling, with additional "natural" lands becoming "urbanised". Some part of the growing city remains "green" and continues to function as an "urbanised" ecosystem. Its characteristics and functioning though become very different, i.e. it is now an "urbanised" ecosystem, where not only the quantitative fluxes of carbon change, but most importantly the qualities of carbon fluxes change significantly (SvirejevaHopkins and Schellnhuber [7]). The estimation of the "green" area depends on the type of urbanisation, for example, the presence or lack of planning for city growth, municipal regulations and laws, the attractiveness of a city for a rural population and "favelas" phenomenon, i.e. the growth of informal settlements in the Third World.

At the present time, the total area of urbanised territories is relatively small, compared to the total territory participating in the GCC, and is estimated to be a little more than $1 \%$ of the total land area (Miller [2]). However, if we take into account the paradoxes of exponential growth so that a factor being negligibly small these days could rapidly become significantly important in the near future, urban territories are good candidates for becoming a leading actor in land use. As compared to our earlier approach, Svirejeva-Hopkins et al [5], that was based on the linear regression of urban area to urban population and statistically predicted an area's dynamics until the year 2020, here we have enriched our database by up to 1248 cities and added the generalised statistics for a dynamic case by implementing the multi-regional demographic model (Svirezhev et al [8]) that allowed us to predict the dynamics of urban areas further into the future, until 2050, for the eight world regions: Economies in Transition (ET); Highly industrialised/Europe (HI); USA, Canada, Australia and New Zealand (UCA); China (Cn); Africa (Afr); Latin America and the Caribbean (LAC); Arab States (Ar); Asia and the Pacific (AsP). Comparing the new estimations of the regional urban area growth to the previous statistical predictor [5], one could note that the estimations of the regional urbanised areas, although qualitatively coinciding in dynamics, differ in their values, i.e. gamma-model predicts higher numbers for UT.

\subsection{Population density}

One of the most important variables when determining the total contribution of urbanised territories to the GCC - at global, regional and local levels - is their area. However, although there are many prognoses in the literature, they are mostly for the population values. Nevertheless, there is one standard 
demographic variable that contains both spatial and population information: population density. It is obvious that if we know the density threshold that determines when a territory becomes urbanised then we may estimate the percent of urbanised area. There is some difficulty, however, in how to define the urban.

After many attempts have been made to find universal definitions, based on density, and none having been totally successful, we have decided to use the "directive" definition of the threshold density when this value is given ad hoc. The point is that to set the exact boundary between urban and rural territories with respect to population density is a difficult task, while the absence of a strong, formal definition generates fuzziness in the boundary. As a result, territories with very low densities are often accounted in national statistics as urban ones. It is important to take into consideration that when we estimate urban areas, we operate within the tails of the sampling distributions, which are as a rule determined with low accuracy; while the situation is not significantly improved if we change from the normal to log-normal distribution of densities.

\subsection{Population density distribution}

Generally speaking, for our purposes, it is more convenient to use another distribution, $p(D)$, where the value $p(D) \Delta D$ is the relative area (percent with respect to the total area, $S_{t}$ ) of such a domain, the population density of which lies between $D$ and $D+\Delta D$. It is obvious that the normalizing condition below must hold:

$$
\int_{0}^{\infty} p(D) d D=1 .
$$

An urbanised area is then defined as:

$$
S_{u}=S_{t} \cdot \int_{D^{*}}^{\infty} p(D) d D .
$$

where $D^{*}$ is the threshold density of population for urbanised territories. The mean population density is equal to:

$$
\hat{D}=N_{t} / S_{t}=\int_{0}^{\infty} D p(D) d D .
$$

If the proportion of a population inhabiting urbanised territories is known $\left(k_{u}\right)$ then:

$$
k_{u} \hat{D}=\int_{D^{*}}^{\infty} D p(D) d D
$$


So, if the distribution $p(D)$ belongs to the class of two-parametric distribution, and its type is known, then there is no problem to estimate the urbanised area if we know the total population, $N_{t}$, the percent of urban population, $k_{u}$, and the density threshold, $D^{*}$, for a given region.

In their classic monograph Kendall \& Stuart [1] have indicated that the spatial distribution of population density for different species (including Homo sapiens) is close to the two-parametric gamma-distribution:

$$
p(D)=\frac{1}{\Gamma(\alpha) \beta}\left(\frac{D}{\beta}\right)^{\alpha-1} \exp (-D / \beta) .
$$

where $\Gamma(\alpha)$ is Euler's gamma-function, $\alpha>0$ determines the form of distribution and $\beta$ is a scale factor, see fig 1. Note that Vaughn [9] has successfully applied this distribution for the description of population densities in a city centre.

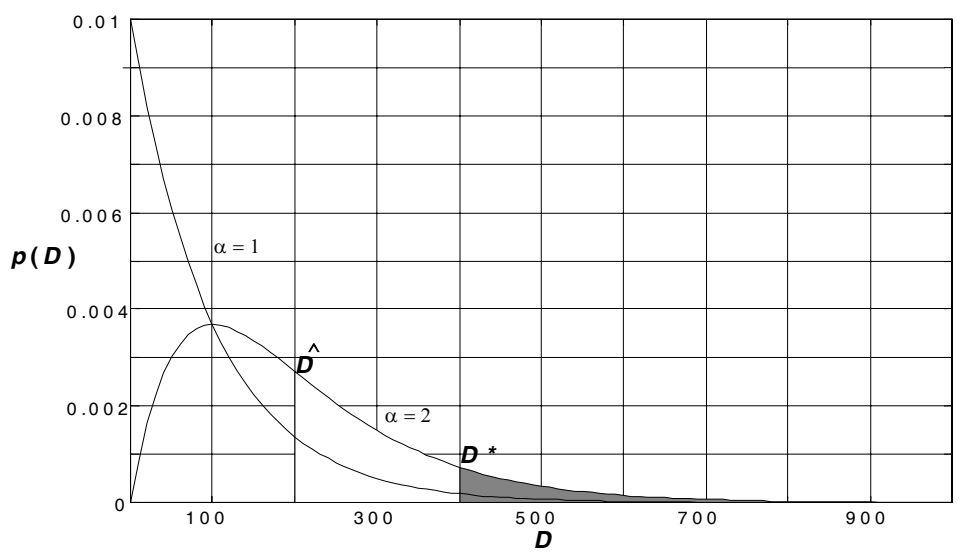

Figure 1: Gamma-distribution as the distribution with density $p(D)$.

Equalities (4) and (5) can be used to estimate these parameters. After such calculations, we obtain:

$$
\hat{D}=\alpha \beta \text { and } k_{u}=\frac{\Gamma(\alpha+1 ; \alpha \lambda)}{\Gamma(\alpha+1)}, \lambda=\frac{D^{*}}{\hat{D}} .
$$

where $\Gamma(\alpha+1 ; \alpha \lambda)$ is the incomplete Euler function. Finally, knowing the parameters $\alpha$ and $\beta$ for each region, the percentage of urbanised territory in this region is calculated as: 


$$
s_{u}=S_{u} / S_{t}=\frac{\Gamma(\alpha ; \alpha \lambda)}{\Gamma(\alpha)} .
$$

For instance, the area of the grey domain in fig. 1 is equal to $S_{u}$.

Hence, as shown above, urbanised areas may be described through the quantiles of this distribution. Since its parameters depend upon such demographic characteristics as the mean population densities and the density threshold corresponding to an urban territory, by the same token we will obtain the dependence of urban area on urban population.

\subsection{Data and demographic prognoses: scenario}

As a scenario we use the UN regional prognoses for the total and urban population with some correction, which was necessary to introduce more accuracy to prognoses of the total regional populations. Therefore we use the statistical data and prognoses for the total country population from UN [10] and [8]. In order to estimate the percentages of urbanised populations for the world's regions, we use data from [10] with some corrections and extrapolations.

\section{Method: Construction of a model for urban areas' growth based on the gamma-distribution}

\subsection{Parametric distribution}

As it was shown in a previous section, In order to construct a functional relationship connecting the area of urbanised territory and its population, we can use some parametric distribution, the parameters of which are some function of urban population, and, possibly, other demographic characteristics.

We assume that the distribution of population density is described by the formula (5). This determines a fraction of the total area populated with a density range between $D$ and $D+\Delta D$. This share is equal to $p(D) \Delta D$. As already shown, the parameters $\alpha$ and $\beta$ are determined by using demographic characteristics.

We describe this further using:

$$
k_{u}=N_{u} / N_{t}=\frac{\Gamma(\alpha+1 ; \alpha \lambda)}{\Gamma(\alpha+1)} \quad \text { and } \quad \hat{D}=N_{t} / S_{t}=\alpha \beta
$$

where $\lambda=D^{*} / \hat{D}$ and $\Gamma(\alpha+1 ; \alpha \lambda)$ is the incomplete Euler function (Ryzhik and Gradstein [3]). Finally, knowing the parameters $\alpha$ and $\beta$ for each region, the share of urbanised territory (in relation to the total area) in this region is calculated as: 


$$
s_{u}=S_{u} / S_{t}=\frac{\Gamma(\alpha, \alpha \lambda)}{\Gamma(\alpha)} .
$$

or, using the various relations for gamma-functions from [3] we obtain:

$$
s_{u}=k_{u}-\frac{(\alpha \lambda)^{\alpha} e^{-\alpha \lambda}}{\Gamma(\alpha+1)} .
$$

If the values of $\lambda$ and $k_{u}$ are known, then the values of $\alpha$ are found as solutions of the functional eqn. $(6$, left). We presume that the values of the density threshold do not change with time, since within each region urban lifestyle, traditions, structure etc. do not change rapidly over the course of considered time. Substituting the inferred values of $\alpha$ into eqn. (9) or eqn. (10), we obtain the relative areas of urbanised territory ( $\%$ of the total regional area) for each region, fig. 2. Considering the dynamics of the relative regional urban areas we can say that regions such as Afr, Ar, $\mathrm{Cn}$ and AsP manifest fast increasing areas of urbanised territory. For instance, urban area in the Africa region will increase 3-4 times during the 80 years (between 1980 and 2050), while, during the same time, the HI, ET and UCA regions will experience insignificant increases in urban area. Fig.3 shows the world dynamics of relative urbanised area. We see that the percentage of the world's urban area in 1985 is about $3 \%$, which is very close to the estimation by Small and Cohen [4].
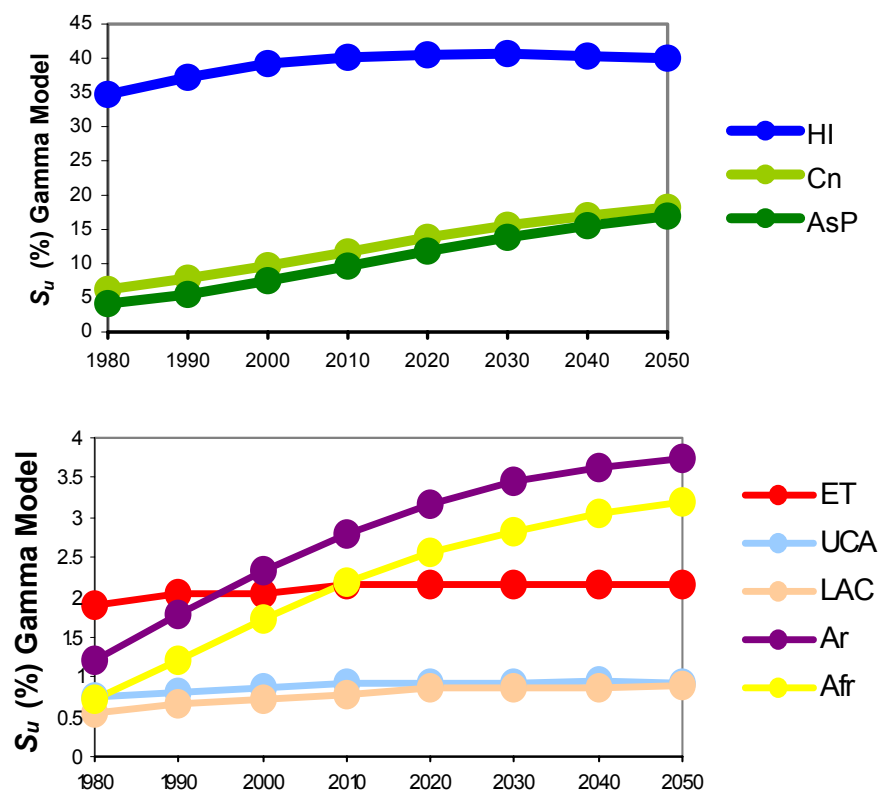

Figure 2: Dynamics of the regional relative urban areas ( $\%$ of the total regional area). 


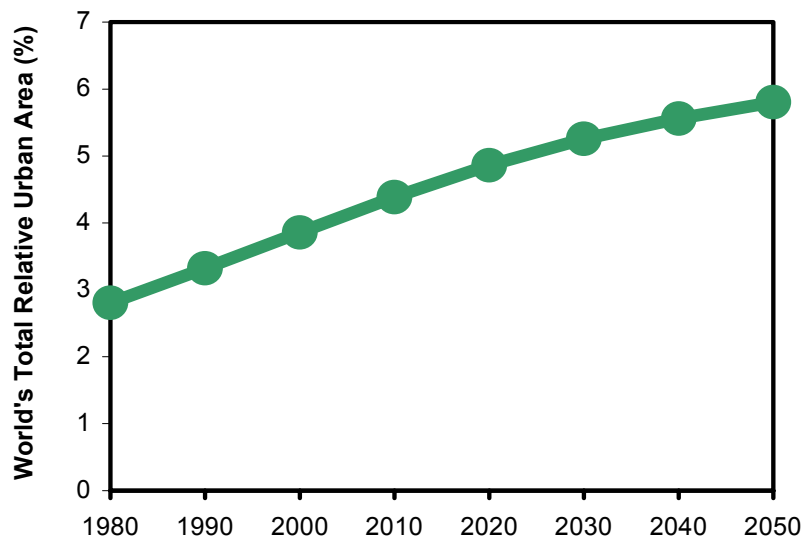

Figure 3: Dynamics of the World relative urban area.

\subsection{Emissions, export and the total carbon balance in urbanised areas}

As the next step we calculate the dynamics of all three carbon flows [7], i.e. emission, export and the total balance, by using data describing the dynamics of an urbanized area obtained by means of the gamma-model and applying the model of non-random cities distribution, i.e. taking into account that cities are not distributed randomly within the region, but are located within the certain biomes. These calculations were performed for the eight World regions (Svirejeva-Hopkins and Schellnhuber [6]), but we will show here the results for the World in fig. 4; where a result of interaction between the annual carbon losses due to land conversion accompanying the process of urbanisation, $d C_{l}(t)$ and the annual export of carbon, $d C_{e}(t)$, is the total carbon balance of the exchange fluxes between the atmosphere and urbanised territory, $d C_{t o t}(t)$.

\section{Discussion}

The World as a whole is an integrator of all-regional tendencies, so let us see what kind of tendencies are observed at the global level.

For the annual emission flow out of the world urbanised territories, fig. 4, we can say that it will slowly increase from $1.12 \mathrm{GtC}$ per year in 1980 up to $1.25 \mathrm{GtC}$ per year in 2005, after which an increasingly accelerated decrease starts, such that by the year 2050 emissions will have fallen to $623 \mathrm{MtC}$. If we compare the emission maximum, $1.25 \mathrm{GtC}$ per year, with the annual emission caused by the process of deforestation, $1.36 \mathrm{GtC}$ per year in 1980, then we can say that the role of urbanised territories in the GCC is of a comparable magnitude as the role of deforestation.

World dynamics of the annual "horizontal" export of carbon out of urbanised territories is the following: there is an almost linear growth from $249 \mathrm{MtC}$ in 

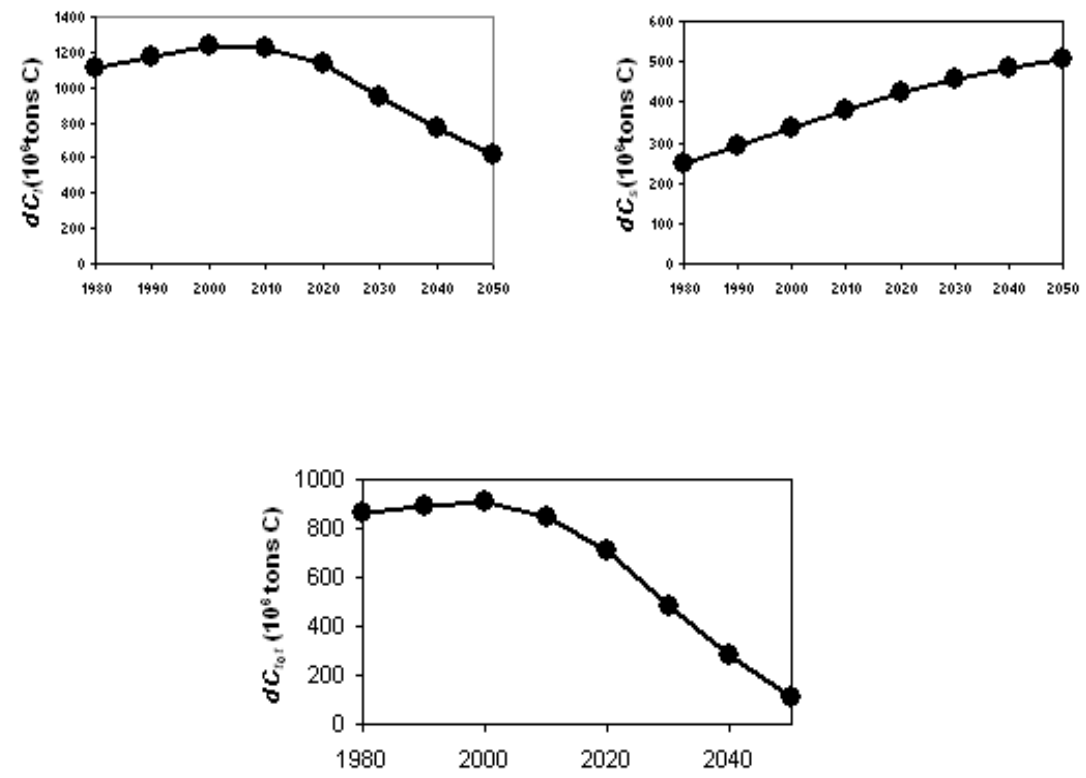

Figure 4: World annual emission of carbon as a result of land conversion (upper left); annual export of organic carbon out of "urbanised" ecosystems into neighbouring territories (upper right) and dynamics of the total carbon (bottom centre).

1980 up to $505 \mathrm{MtC}$ in 2050 , i.e., during those seventy years export increases two fold. Basically, the transport power of urbanised territories is comparable to the amount of carbon transported by rivers into the ocean (196-537MtC per year, [8]).

Finally, the total carbon balance being almost constant until 2000, then starts decreasing at an almost constant rate. If its maximal value in 2000 is $905 \mathrm{MtC}$, then by 2050 this value has fallen to $118 \mathrm{MtC}$, i.e., by almost eight times. By extrapolating this graph into the future, we can say that by the end of the XXI century, the total carbon balance will be equal to zero, and may even be negative (note that we are talking only about land conversion here). The picture is quite different for the regional dynamics, for example China and ASP regions are continuing to be major sources, while HI region has switched from the source state to the sink one around 2006. However, it is necessary to note that the formation of "sinks" in urbanised territories is accompanied by the appearance of "sources" in other locations. In general, this situation is functionally similar to the case when rivers transport organic carbon to other locations, where it is then decomposed and emitted into the atmosphere. 


\section{Conclusions}

Gamma-model infers dynamics of urbanisation that are very similar to realistic one. The reason for this is that this dynamics is, on the one hand, a result of the generalization of concrete statistical data, while on the other, is a consequence of statistical extrapolation to a near future. It is natural that the use of a model based on the two-parametric gamma-distribution is more attractive then using statistical model, since these parameters are integral indicators that combine demographic characteristics, such as the threshold density, which in turn is connected with life quality and also with a city structure. Therefore, as a future development, by changing the threshold density in accordance with some scenarios, we can study the dynamics of urbanised areas as a function of such scenarios. To estimate the role of UT in the global balance of carbon, we have calculated the total balance of carbon between the atmosphere and urbanised territories, when UT act either as sink or source of carbon. When the growth of UT in a given region happens faster, then the shift towards a "source" state is greater. The "through-pumping" of atmospheric carbon through an "urbanised" ecosystem shifts its state to a "sink". This depends on the green area: the larger it is, the greater is the shift towards a "sink" state. The model forecasts that the peak of urbanisation will happen in a decade. In this light it becomes especially important to consider dynamics of UT and to include urbanisation as a component in the Global Carbon Models.

\section{References}

[1] Kendall, M. G. \& Stuart, A., The Advanced Theory of Statistics, v. 1.2. Academic Press, New York. 1958.

[2] Miller, G.T., Living in the Environment, 6th ed. Woodswort, Belmont, CA., 1988.

[3] Ryzhik, I. S. \&. Gradstein, I. M., Tablicy intiegralov, sum, riadow $i$ proizvedenij, Nauka, Moskva, (in Russian), 1971.

[4] Small, C. \& Cohen, J.E., Continental physiography, climate and the global distribution of human population. Proc. of the International Symposium on Digital Earth. Chinese Academy of Science, Beijing: 965-971, 1999.

[5] A. Svirejeva-Hopkins, H.-J.Schellnhuber, V.L. Pomaz, Urbanised territories as a specific component of the Global Carbon Cycle. Ecological Modelling, 173, pp. 295-312, 2004.

[6] A. Svirejeva-Hopkins \& H.-J.Schellnhuber, Urbanised territories as a specific component of the Global Carbon Cycle. PIK Report No. 94, ISSN 1436.0179, 2005.

[7] Svirejeva-Hopkins, A. \& Schellnhuber H.-J., Modelling carbon dynamics from urban land conversion: fundamental model of city in relation to a local carbon cycle. Carbon Balance and Management, 1:8, 15 August. http://www.cbmjournal.com 2006. 
[8] Svirezhev, Yu. M. et al. New version of the Moscow Global Biosphere Model. PIK Core Project BBM/Gaia. Final scientific report. PotsdamInstitut für Klimafolgenforschung, Potsdam - Moscow: 834pp., 1997.

[9] Vaughn, R., Urban Spatial Traffic Patterns, Pion, London, 1987.

[10] United Nations (UN), The state of the World Cities 2001. Centre for Human Settlements, UNCHS: 121pp., 2001. 